\title{
Association of dysbindin expression with individualized postoperative prognosis and chemotherapy benefit among patients with gastric adenocarcinoma
}

\author{
Hao Qian ${ }^{1,2 \#, ~ X i a o h u i ~ L v #, ~ Q i y i n g ~ S o n g 4 \#, ~ R u j u a n ~ S u}{ }^{5 \#, ~ T i a n y u ~ X i e 4, ~ D i ~ W u ~}{ }^{4}$, Rongyan Chang2 ${ }^{2}$ Lubin \\ Chen $^{2}$, Yanling Yang 6 , Yong Chen ${ }^{6}$, Xinxin Wang ${ }^{*}$, Yi Ru ${ }^{7^{*}}$, Lei Shang ${ }^{*}$, Xin Guo ${ }^{2,4 \bowtie *}$ \\ 1. Department of Health Statistics, School of Public Health, Fourth Military Medical University \\ 2. Department of Endoscopic Surgery, Xijing Hospital, Fourth Military Medical University. \\ 3. Department of Gynecology and Obstetrics, Xijing Hospital, Fourth Military Medical University. \\ Department of General Surgery, Chinese PLA General Hospital. \\ Department of Oncology, Second Affiliated Hospital of Xi'an Jiaotong University. \\ Department of Hepatobiliary Surgery, Xijing Hospital, Fourth Military Medical University. \\ Department of Biochemistry and Molecular Biology, Fourth Military Medical University. \\ \#Authors share co-first authorship. \\ *Authors share co-senior authorship. \\ $\triangle$ Corresponding author: Xin Guo, Address: FMMU Xijing Hospital, Changle 169, Xi'an, Shaanxi Province, China, 710054. Tel.: 86-029-84734091; Fax: 86-029-84734092; \\ E-mail: guoxin0425@sina.com. \\ (C) The author(s). This is an open access article distributed under the terms of the Creative Commons Attribution License (https://creativecommons.org/licenses/by/4.0/). \\ See http://ivyspring.com/terms for full terms and conditions.
}

Received: 2021.03.17; Accepted: 2021.08.28; Published: 2021.09.21

\begin{abstract}
Background: The current model for predicting prognosis and chemotherapy response of patients with gastric adenocarcinoma is the TNM staging system, which may lack adequate accuracy and evaluations of molecular features at the individual level. We aimed to develop a prediction model to assess the individualized prognosis and responsiveness to fluorouracil-based adjuvant chemotherapy.

Method: This retrospective study concluded 2 independent cohorts of patients with GAC. The expression of dysbindin was quantified and evaluated the association with the overall survival for GAC patients. A prediction model for postoperative overall survival was generated and internally and externally validated. The interaction between dysbindin expression and PACT was detected in advanced GAC patients.

Results: Of the 637 patients enrolled in the study, 425 were men (66.7\%) with a mean (SD) age of 59.79 (9.81) years. High levels of dysbindin expression predicted a poor prognosis in patients with GAC. Multivariate analysis demonstrated dysbindin expression was an independent prognostic predictor of overall survival in the test, validation and combined cohorts. A prognostic predictive model incorporating age, dysbindin expression, pathological differentiation, Lauren's classification and the TNM staging system was established. This model had better predictive accuracy for overall survival than the traditional TNM staging system and was internally and externally validated. More importantly, advanced GAC patients with low dysbindin expression were likely to benefit from fluorouracil-based PACT.

Conclusion: The risk stratification model incorporating dysbindin expression and TNM staging system showed better predictive accuracy. Advanced GAC patients with low dysbindin expression revealed better response of fluorouracil-based adjuvant chemotherapy.
\end{abstract}

Key words: dysbindin; overall survival; TNM stage; chemotherapy benefit

\section{Introduction}

Although the incidence and mortality rates of gastric adenocarcinoma (GAC) have declined in recent decades, in East Asian countries such as China, GAC remains the second most commonly diagnosed malignancy and the second leading cause of cancer- related death [1,2]. Currently, radical gastrectomy is generally advised as the only curative treatment for GAC [3]. Unfortunately, the high rates of postoperative recurrence and metastasis results in poor overall survival (OS), making it critical to 
consider adjuvant treatments $[4,5]$. However, recent studies have indicated that GAC patients who received PACT based on fluorouracil regimens have only slightly improved OS rates, making the value of adjuvant chemotherapy controversial [6-9]. Moreover, similar regimens of adjuvant chemotherapy in GAC patients with the same TNM stage result in a wide variety of clinical outcomes [10, 11]. The current strategy for assessing risk stratification and prognosis is the TNM staging system, which may lack sufficient accuracy, especially in stage II and III GAC patients $[12,13]$. Consequently, an accurate risk stratification for GAC patients is warranted to improve the potential individual benefits obtained from postoperative chemotherapy.

Dysbindin, encoded by the dystrobrevin binding protein 1 gene, is a protein that functions as a component of the BLOC- 1 complex, which is required for the normal biogenesis of lysosome-related organelles [14]. In cancers, dysbindin is a novel oncoprotein that regulates the phosphorylation of the PI3K-Akt and ERK signaling pathways, which can lead to tumorigenesis and chemotherapy resistance $[15,16]$. In pancreatic ductal adenocarcinoma and ovarian cancer, dysbindin serves as an independent factor for prognosis and promoted invasion and metastasis [16, 17]. However, the association of dysbindin expression and GAC remains unknown, and the underlying mechanism by which dysbindin acts in GAC requires further investigation.

In this study, we describe a novel model based on the TNM staging system and dysbindin expression that can be used to assess the individual risk and OS probability of patients with GAC. Furthermore, we aimed to explore whether the dysbindin expression status could be used to identify patients with advanced GAC who might sensitive to fluorouracilbased PACT.

\section{Materials and Methods}

\section{Institutional Review Board Statement}

The Medical Ethics Committee at each involved Chinese institutional medical center (Xijing Hospital, Fourth Military Medical University and the Chinese People's Liberation Army General Hospital) approved this study. All patients signed the informed consent form before their resected tissues were used.

\section{Patients and specimens}

In this study, a test cohort of 375 consecutive GAC patients (male: female $=256: 119$ ) was recruited from the Chinese People's Liberation Army General Hospital from October 2012 to May 2015 for model establishment and internal validation. An additional cohort of 262 consecutive patients (male: female
$=169: 93$ ) with the same diagnosis at Xijing Hospital from January 2013 to February 2015 was enrolled for external validation. The inclusion criteria were as follows: 1. the diagnosis of histologically confirmed GAC; 2 . treatment with standard radical gastrectomy; 3. patients with advanced stage GAC were administered postoperative fluorouracil-based adjuvant chemotherapy for at least 4 cycle [18]. Follow-up data needed to be available. The exclusion criteria were as follows: 1 . preoperative anticancer treatments including chemotherapy, radiotherapy, immunotherapy or other cytotoxic therapy, and postoperative anticancer treatments in addition to routine chemotherapy; 2 . the existence of distant metastasis; and 3. postoperative death due to complications.

The detailed clinicopathological characteristics of each patient, including gender, age, tumor size, tumor location, pathological differentiation, Lauren's classification and the TNM staging system were retrospectively collected. Pathological differentiation was classified as well, moderate, poor and undifferentiated according to the World Health Organization's gastric cancer treatment guidelines [19]. The clinical staging was determined according to the $7^{\text {th }}$ edition of the American Joint Committee on Cancer (AJCC) and the International Union Against Cancer tumor-node-metastasis (TNM) staging system [20]. OS refers to the time from the operation to the last follow-up or death. The median (range) follow-up time was 30.454 (2.8-87.2) months for the test cohort and 28.352 (3.9-88.5) months for the validation cohort. Data were analyzed between January 2020 and June 2020.

\section{Immunohistochemistry and evaluation of immunostaining}

GAC and adjacent noncancerous tissues were obtained immediately after gastrectomy. Adjacent noncancerous tissues were defined as specimens adjacent to the margins of GAC (within $5 \mathrm{~cm}$ ). Before tissue microarray construction and immunohistochemistry, all specimens were sliced into $4 \mu \mathrm{m}$ sections and stained by hematoxylin-eosin for accurate histological confirmation and selection of appropriate representative regions for each tissue. Immunohistochemical staining results were evaluated by two independent gastrointestinal pathologists who were blind to the study, and their results were averaged. Dysbindin expression was assessed with immunohistochemically staining according to the method described previously $[17,21]$. The immunohistochemical staining scores were determined as described previously by the following formula: intensity score $\times$ proportion score [22-24]. Briefly, the 
indicated staining scores were applied for the corresponding intensities ( 0 , no staining; 1 , light yellow; 2, yellow brown; 3, strong brown color), and the indicated scores were applied for the corresponding proportions of positive tumor cells $(0$, $0 \%$ positive tumor cells; $1,0 \%-10 \%$ positive tumor cells; $2,10 \%-50 \%$ positive tumor cells; $3,50 \%-100 \%$ positive tumor cells). The final immunoreactivity score (IS) for grouping was the product of the staining area score and the staining intensity. For the statistical analysis, the scores were grouped in two categories: scores of 0-3 were considered as low expression and 4-9 as high expression.

\section{Prediction model development and validation}

Univariate and multivariate regression analyses were used to estimate the hazard ratio (HR) with 95\% confidence intervals (CI) and determine the independent prognostic factors. The predictive model was constructed based on the known clinical prognostic factors and availability in the test cohort based on independent risk factors [25]. After the test of Cox proportional hazards assumption, this model was implemented into the nomogram for predicting 3- and 5-year overall survival in GAC patients after surgery.

The predictive performance indicators of the nomogram include discrimination and calibration. Firstly, internal validation of the nomogram was performed with one thousand bootstrap resamples from the test cohort. The calibration curves were used to evaluate the goodness of fit between the predicted probabilities and observed outcomes. Secondly, the performance was further verified in the validation cohort and combined cohort to verify the applicability of the nomogram to other populations.

\section{Clinical use}

In addition to the above verification methods, decision curve analysis (DCA) was further performed to compare the clinical usefulness and net benefit of the nomogram with those of the TNM staging system. It is generally considered that models with higher net benefit rates within a specific threshold range are more clinically useful [26].

\section{Statistical analysis}

The continuous data are reported as the means \pm SDs, and the differences between the cohorts were analyzed using independent-sample, unpaired, 2-tailed $t$ tests or Mann-Whitney $H$ tests, as appropriate. Categorical data are presented as proportions and percentages and were evaluated using the chi-square test or Fisher's exact test. Overall survival of patients' subgroups was compared by the Kaplan-Meier survival curve with the log-rank test.
Univariate and multivariate regression analyses were applied to estimate the hazard ratio (HR) with 95\% confidence intervals (CI) and identify the independent prognostic factor by Cox proportional hazards models. Interactions between the dysbindin expression and postoperative adjuvant chemotherapy treatment (PACT) were also detected by the Cox model. All statistical analyses were conducted with SPSS software (SPSS 26.0), RStudio software (RStudio, 1.2.5033) with R soft packages of "rms", "time ROC", and "stdca" and GraphPad Prism (GraphPad Prism, 8.1.244). Differences with two-sided $\mathrm{P}<0.05$ were considered statistically significant. All authors had access to the study data and reviewed and approved the final manuscript.

\section{Results}

\section{Participants}

Our goal was to investigate whether dysbindin expression could serve as a prognostic and predictive indicator to identify patients at high risk and those likely to respond to chemotherapy. To this end, we conducted this study and the flow diagram is shown in Supplementary Fig. 1. The test cohort included of 375 consecutive patients, of whom 256 (68.3\%) were men, with a median (interquartile range [IQR]) age of 57 (51-66) years. The validation cohort included of 262 consecutive patients, of whom 169 were men, with a median (interquartile range [IQR]) age of 58 (50-65) years. The clinicopathological characteristics of the patients in both cohorts were similar and given in Supplementary Table 1.

\section{Association of dysbindin expression with clinicopathological characteristics}

Dysbindin expression was immunohistochemically detected in 637 patients with GAC. Dysbindin expression was high in GAC tissues but low or absent in adjacent noncancerous tissues (Fig. 1). GAC tissues were classified into two groups according to dysbindin expression levels. In the test cohort, 251 specimens (66.9\%) had high expression levels of dysbindin, and 124 (33.1\%) had low levels. In the validation cohort, 168 specimens $(64.1 \%)$ had high expression levels of dysbindin, and 94 (35.9\%) had low levels. In addition, no significant correlation was detected between dysbindin expression and the clinicopathological characteristics of patients with GAC, which are shown in Supplementary Table 2.

\section{Association of dysbindin expression and the prognosis of patients with gastric adenocarcinoma}

In order to explore the prognostic value of dysbindin in patients with GAC, we performed 
Kaplan-Meier survival curves to compare the OS of patients with different levels of dysbindin expression. In the test cohort (HR, 2.516; 95\% CI, 1.890-3.349; $\mathrm{P}<0.0001)$, validation cohort $(\mathrm{HR}, 3.749$; 95\% CI, 2.659-5.286; $\mathrm{P}<0.0001)$ and combined cohort ( $\mathrm{HR}$, 2.947; 95\% CI, 2.366-3.671; $\mathrm{P}<0.0001)$, patients with high dysbindin expression had poorer OS than those with low dysbindin expression (Supplementary Fig. 2).

Then, we performed a stratified analysis of patients with GAC according to their clinicopathological characteristics. In test, validation and combined cohorts, patients with high dysbindin expression both had shorter OS time than patients with low dysbindin expression in AJCC stage I, II and III (Supplementary Fig. 3). Furthermore, dysbindin expression remained a powerful prognostic predictor after stratification by age, gender, tumor size, tumor location, Lauren's classification, pathological differentiation, $\mathrm{T}$ stage and $\mathrm{N}$ stage (Supplementary Fig. 4-6).

Finally, as shown by univariable analysis, patients with high expression levels of dysbindin were associated with significantly poorer OS (Table 1). Furthermore, multivariate Cox regression analysis revealed that dysbindin expression and the TNM staging system were both identified as the independent prognostic factors in GAC patients (Table 1 and Supplementary Table 3-4). Hence, dysbindin expression may serve as a reliable and independent and dangerous prognosticator of OS for patients after GAC surgery.

\section{Prognostic power for dysbindin expression and the TNM staging system}

After the expression of dysbindin and the TNM staging system were confirmed as two independent prognostic risk factors, we then tried to evaluate whether integrating the dysbindin expression and the current TNM staging system will improve the prognostic predictive ability. As shown in the Fig. 2 and Supplementary Table 5, the AUCs of dysbindin expression were lower than those of the TNM staging system. However, the integration of dysbindin expression and the TNM staging system showed better prognostic accuracy for 3- and 5- year OS (test cohort: $0.765,0.774$; validation cohort: $0.832,0.889$; combined cohort: $0.792,0.814)$ than the TNM staging system, dysbindin expression or any other clinicopathological characteristics alone. These results suggest that dysbindin expression may complement the TNM staging system in the prognostic prediction after surgery.

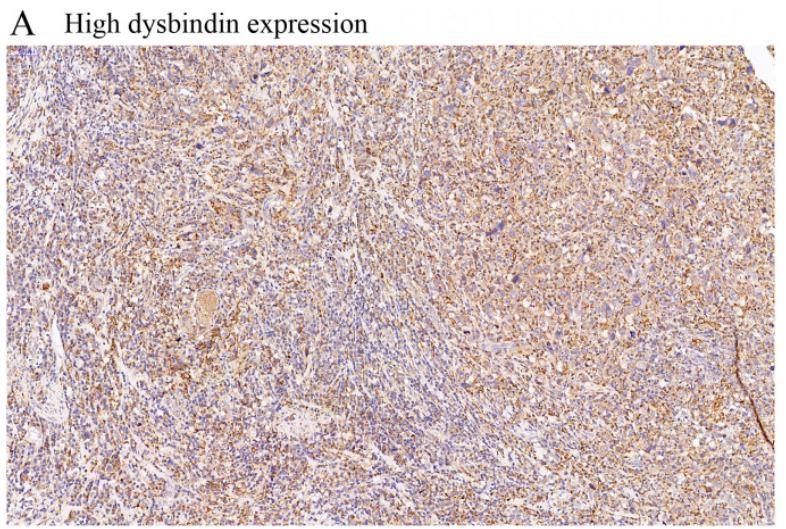

B High dysbindin expression

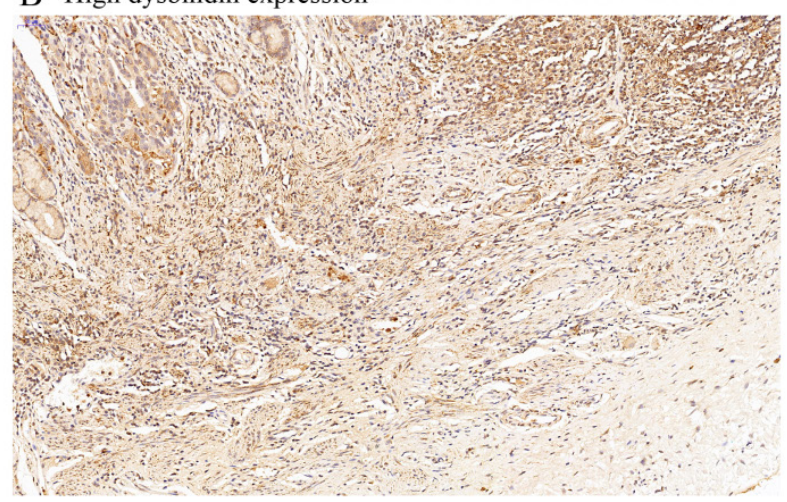

C Low dysbindin expression

D Low dysbindin expression
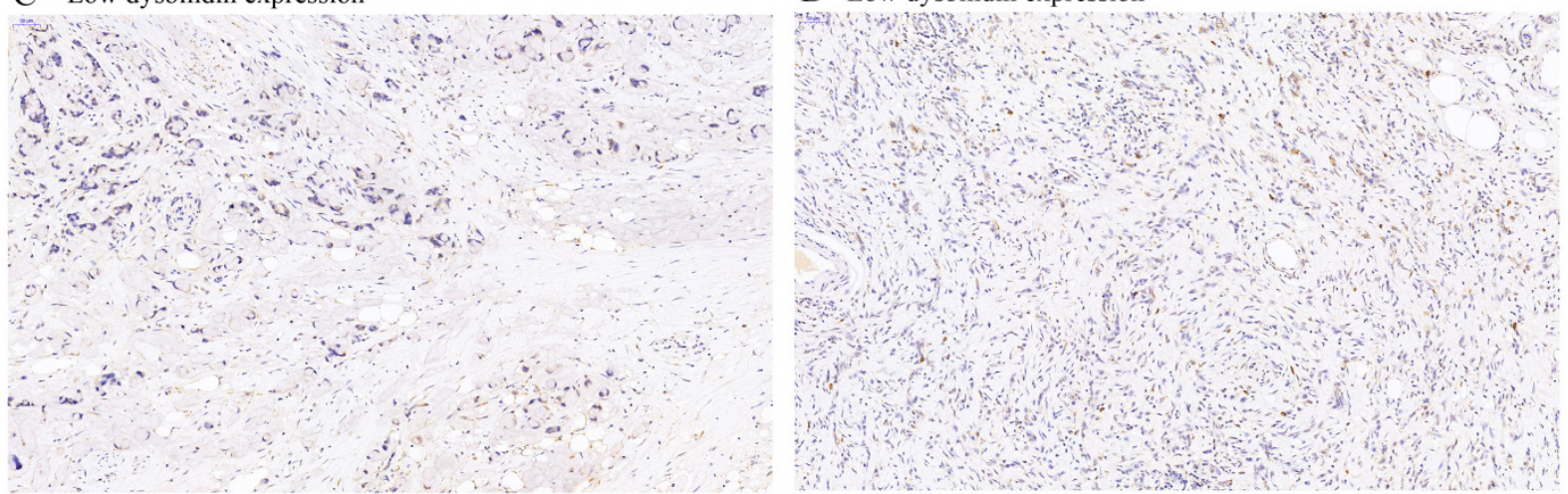

Figure 1. The expression levels of dysbindin in GAC tissues. A and C, Representative staining images of dysbindin high expression. B and D, Representative staining images of dysbindin low expression. All specimens were stained by immunohistochemistry assays with an original magnification of $\times 200$. 
A

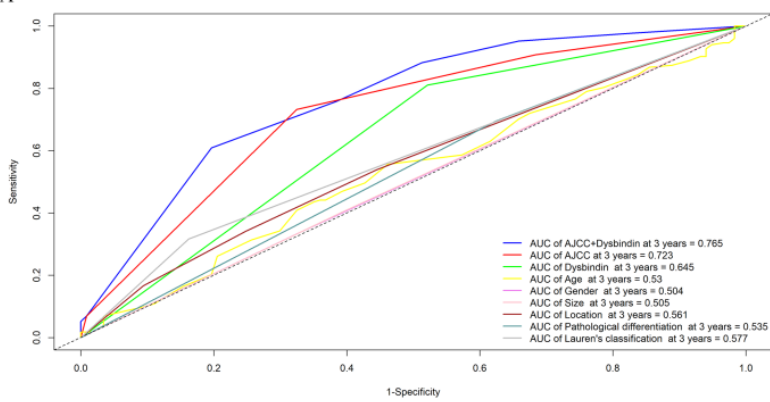

B

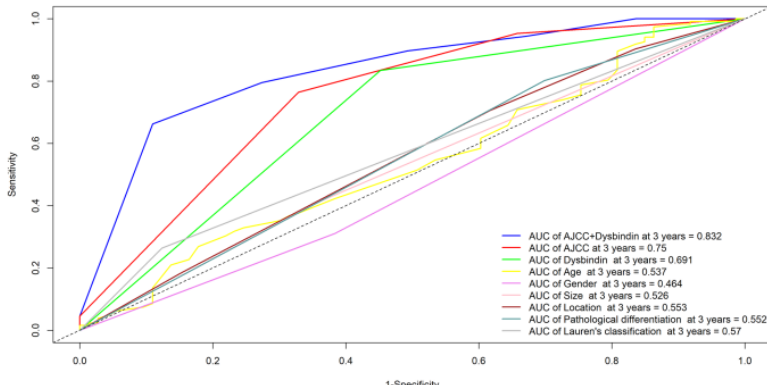

C

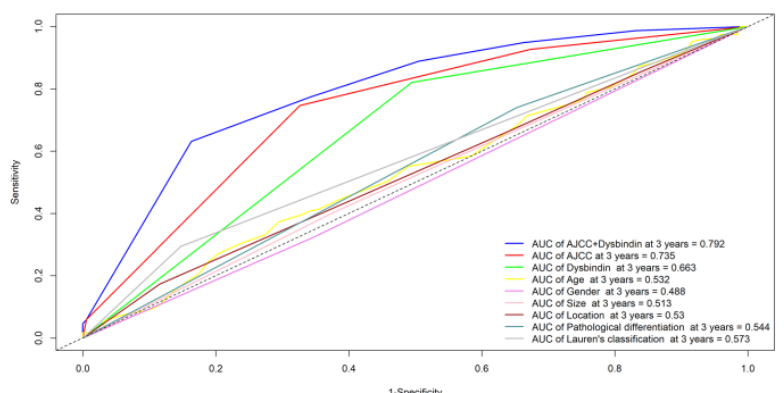

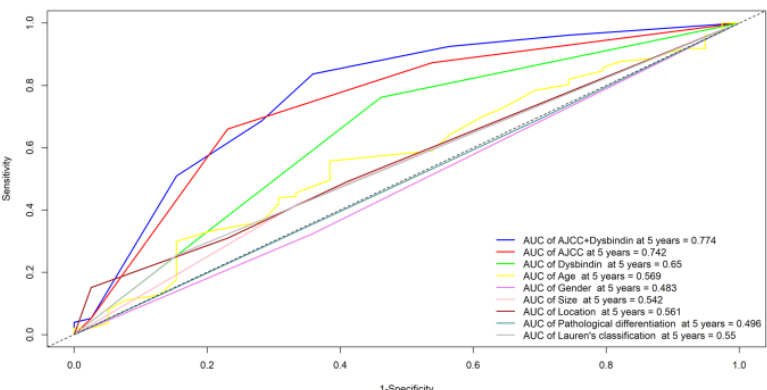
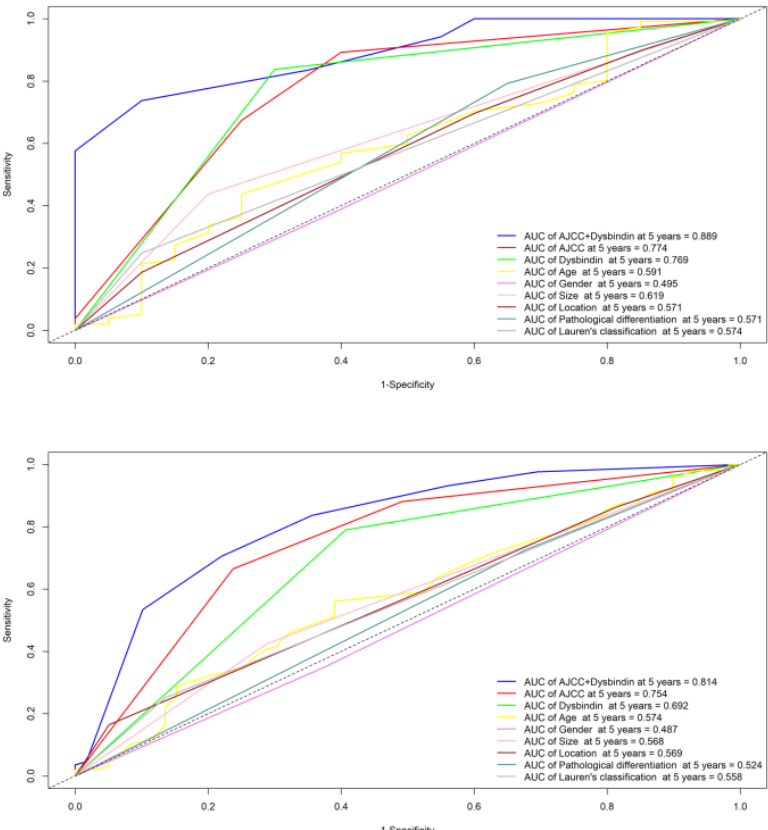

Figure 2. Time-dependent receiver operating characteristic (ROC) curves for assessing dysbindin expression, TNM stage and clinicopathological characteristics as predictors of 3- and 5-year OS in different cohorts. A, the 3-and 5-year ROC curves in test cohort. B, the 3- and 5-year ROC curves in validation cohort. C, the 3- and 5-year ROC curves in combined cohort. The AUC value of each predictor was shown in the figure.

\section{Development and assessment of an individualized prognostic prediction nomogram}

To establish a more effective prognostic model, we constructed a Cox model on the basis of the independent prognostic risk factors and several known clinical prognostic factors. Included covariates were age, TNM staging system, dysbindin expression, Lauren's classification, pathological differentiation. The test of Cox proportional hazards assumption of the model showed that each covariates test and global test were $\mathrm{P}>0.05$, which did not violate the assumption of proportional hazard (Supplementary Fig. 7 and Supplementary Table 6). Then, the Cox model was implemented into the nomogram to predict the overall survival at 3 and 5 years for GAC patients after surgery. Thus, a nomogram incorporating age, pathological differentiation, Lauren's classification, dysbindin expression and the TNM staging system was developed (Fig. 3).
Table 1. Univariate and multivariate Cox regression analysis of patients with GAC in test cohort

\begin{tabular}{|c|c|c|c|c|c|}
\hline \multirow[t]{3}{*}{ Factors } & \multirow[t]{3}{*}{ No } & \multicolumn{4}{|l|}{ Overall survival } \\
\hline & & \multicolumn{2}{|l|}{ Univariate analysis } & \multicolumn{2}{|c|}{ Multivariate analysis } \\
\hline & & HR $(95 \% \mathrm{CI})$ & $P$ value & $\mathrm{HR}(95 \% \mathrm{CI})$ & $P$ value \\
\hline Dysbindin expre & ession & & $<0.001$ & & $<0.001$ \\
\hline Low & 124 & 1 (Reference) & & 1 (Reference) & \\
\hline High & 251 & $2.547(1.811-3.581)$ & & $2.544(1.803-3.590)$ & \\
\hline Gender & & & 0.382 & & \\
\hline Male & 256 & 1 (Reference) & & & \\
\hline Female & 119 & $1.142(0.848-1.538)$ & & & \\
\hline Age (years) & & & 0.007 & & 0.125 \\
\hline$\leq 60$ & 229 & 1 (Reference) & & 1 (Reference) & \\
\hline$>60$ & 146 & $1.017(1.005-1.030)$ & & $1.010(0.997-1.022)$ & \\
\hline Size $(\mathrm{cm})$ & & & 0.591 & & \\
\hline$\leq 4$ & 225 & 1 (Reference) & & & \\
\hline$>4$ & 150 & $1.082(0.811-1.443)$ & & & \\
\hline Tumor location & & & 0.558 & & \\
\hline Cardia & 75 & 1 (Reference) & & & \\
\hline Body & 61 & $1.074(0.667-1.729)$ & & & \\
\hline Antrum & 189 & $0.971(0.662-1.425)$ & & & \\
\hline Whole & 50 & $1.310(0.802-2.138)$ & & & \\
\hline Pathological diff & ferent & tiation & 0.113 & & \\
\hline Well + moderate & 128 & 1 (Reference) & & & \\
\hline $\begin{array}{l}\text { Poor }+ \\
\text { undifferentiated }\end{array}$ & 247 & $1.284(0.942-1.751)$ & & & \\
\hline
\end{tabular}




\begin{tabular}{|c|c|c|c|c|c|}
\hline \multirow[t]{3}{*}{ Factors } & \multirow[t]{3}{*}{ No } & \multicolumn{4}{|l|}{ Overall survival } \\
\hline & & \multicolumn{2}{|l|}{ Univariate analysis } & \multicolumn{2}{|c|}{ Multivariate analysis } \\
\hline & & HR $(95 \%$ CI $)$ & $P$ value & $\mathrm{HR}(95 \% \mathrm{CI})$ & $P$ value \\
\hline \multicolumn{3}{|c|}{ Lauren's classification } & 0.126 & & \\
\hline Intestinal type & 272 & 1 (Reference) & & & \\
\hline Diffuse type & 103 & $1.282(0.933-1.762)$ & & & \\
\hline TNM stage & & & $<0.001$ & & $<0.001$ \\
\hline I & 58 & 1 (Reference) & & 1 (Reference) & \\
\hline II & 91 & $1.550(0.911-2.638)$ & & $1.440(0.844-2.457)$ & \\
\hline III & 213 & $3.158(1.971-5.059)$ & & $2.873(1.787-4.619)$ & \\
\hline IV & 13 & $\begin{array}{l}12.035 \\
(5.962-24.294)\end{array}$ & & $12.351(5.973-25.543)$ & \\
\hline
\end{tabular}

The performance of the nomogram was verified by discrimination and calibration. This new prediction model showed adequate accuracy for the prediction of OS at 3 and 5 years in patients after GAC operation, supported by the calibration curves which revealed good consistency between predicted and observed outcomes (Supplementary Fig. 8) in the test cohort, validation cohort and combined cohort. The results of the comparation between the nomogram and the TNM staging system showed that the C-index of the nomogram (test cohort, 0.720 ; validation cohort, 0.718; combined cohort, 0.719) was significantly higher than those of the TNM staging system (test cohort, 0.646; validation cohort, 0.632; combined cohort, 0.641) (Supplementary Table 7). The timedependent ROC of the nomogram also showed better prediction accuracy than that of the TNM staging system (Supplementary Fig. 9). Furthermore, the same results were obtained for each time period in the time-dependent AUC analysis in test and combined cohorts (Supplementary Fig. 10). These results suggest that the novel nomogram had a higher predictive ability than the traditional TNM staging system.

Finally, we also performed decision curve analysis (DCA) to assess the clinical value of this nomogram. As shown in Supplementary Fig. 11, the net benefit rate of the nomogram is better than that of the TNM stage at 3- and 5-year with a large threshold $(\mathrm{Pt})$ range (0.2-0.7). In addition, the DCAs of the validation cohort and combined cohort also showed that the nomogram had a higher net benefit than that of the TNM staging system, regardless of whether it was evaluated at 3-year or 5-year.

\section{Correlation between dysbindin expression and postoperative adjuvant chemotherapy benefit}

Although several classic clinical trials have indicated that patients with GAC derive a survival benefit from postoperative adjuvant chemotherapy, the chemotherapy response varies widely among patients with stage II or III GAC. Thus, we further investigated the correlation between dysbindin expression and the benefit derived from chemotherapy. An interaction test showed that patients with stage II GAC benefited more from adjuvant chemotherapy if they had low dysbindin expression levels than if they had high expression levels (HR, 0.3526; 95\% CI, 0.1152-1.0790; $\mathrm{P}=0.0321$ for interaction). However, patients with stage III GAC showed no significant differences between patients with high dysbindin expression and those with low dysbindin expression (HR, 0.5056; 95\% CI, $0.2677-0.9547 ; \mathrm{P}=0.5720$ for interaction) in Table 2. The Kaplan-Meier survival curves for patients with stage II or stage III GAC, which comprehensively compared the patients with low and high dysbindin levels stratified according to treatment stratification, were shown in Supplementary Fig. 12. These results demonstrated that stage II patients with low level of dysbindin expression and all stage III patients could benefit more from fluorouracil-based postoperative adjuvant chemotherapy.

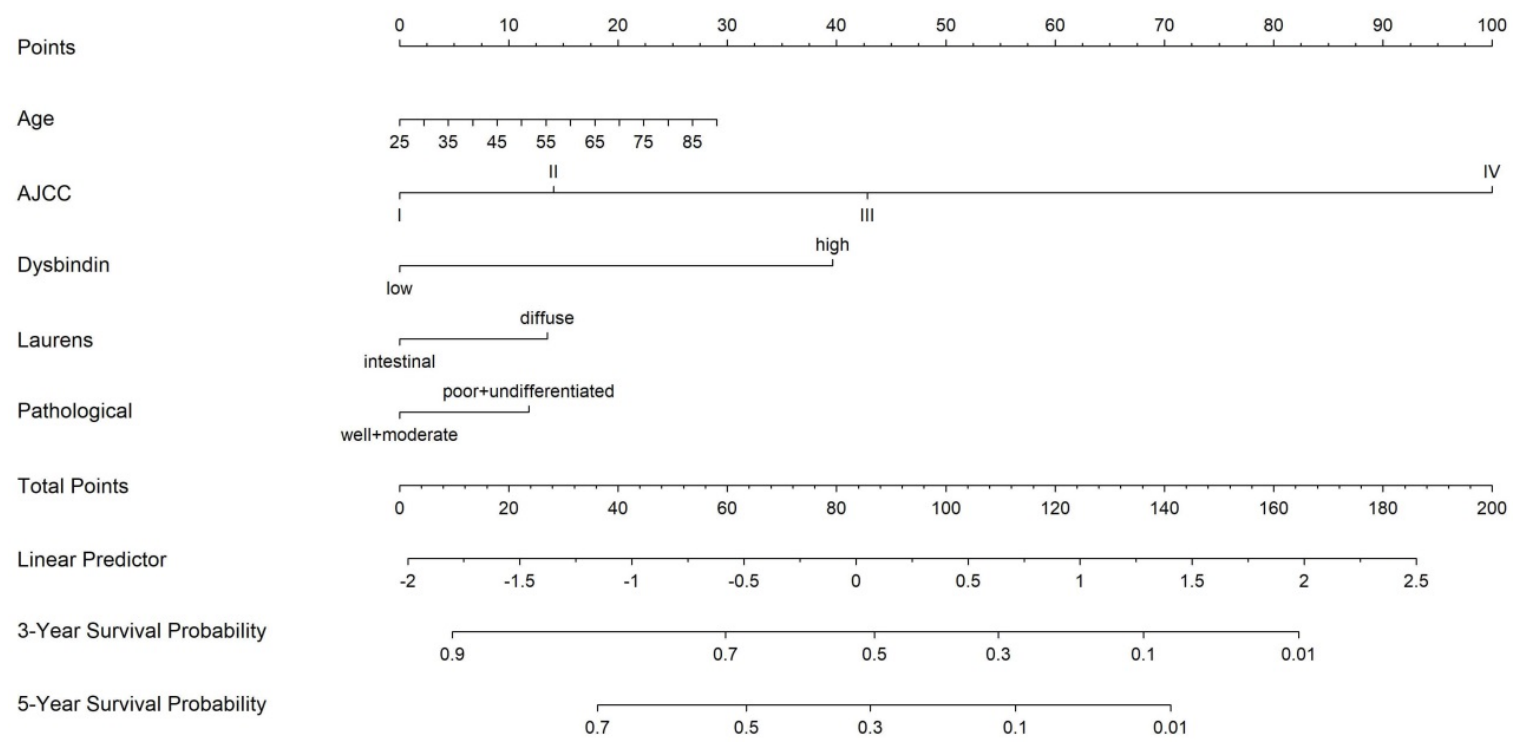

Figure 3. A nomogram to predict 3- and 5-year overall survival rates after D2 gastrectomy for GAC patients. The nomogram is used by summing the points identified on the point scale for each variable. The total points projected on the bottom scales indicate the probability of 3- and 5-year OS. 
Table 2. Treatment interaction with dysbindin expression for overall survival

\begin{tabular}{|c|c|c|c|c|}
\hline $\begin{array}{l}\text { Dysbindin } \\
\text { expression }\end{array}$ & $\begin{array}{l}\text { Chemo- } \\
\text { therapy }\end{array}$ & $\begin{array}{l}\text { No chemo- } \\
\text { therapy }\end{array}$ & HR (95\% CI) & $\begin{array}{l}\text { P value for } \\
\text { interaction }\end{array}$ \\
\hline \multicolumn{5}{|c|}{ AJCC stage II GAC } \\
\hline $\begin{array}{l}\text { High expression } \\
\text { group }(n=93)\end{array}$ & 48 & 45 & $0.7052(0.3999-1.2430)$ & 0.0321 \\
\hline $\begin{array}{l}\text { Low expression } \\
\text { group }(n=54)\end{array}$ & 30 & 24 & $0.3526(0.1152-1.0790)$ & \\
\hline \multicolumn{5}{|c|}{ AJCC stage III GAC } \\
\hline $\begin{array}{l}\text { High expression } \\
\text { group }(\mathrm{n}=258)\end{array}$ & 141 & 117 & $0.6608(0.4861-0.8984)$ & 0.5720 \\
\hline $\begin{array}{l}\text { Low expression } \\
\text { group }(\mathrm{n}=121)\end{array}$ & 50 & 71 & $0.5056(0.2677-0.9547)$ & \\
\hline
\end{tabular}

\section{Discussion}

GAC is a highly heterogeneous disease with various difference in clinical outcomes. Thus, accurate prognostic evaluation is very important when determining the appropriate treatment. The traditional model for prognostic risk stratification and postoperative treatment determination in patients with GAC is based on the TNM staging system. However, this existing model has limitations due to the lack of sufficient accuracy even among patients with the same TNM stage. Recently, treatment based on molecular tumor features has offered an increasingly promising approach to therapy decisionmaking in patients with lung cancer and colorectal cancer [27-30]. In our study, we identified dysbindin expression as the independent prognostic predictive factor in patients with GAC by Cox analysis. The ROC curves showed that the integration of dysbindin expression and TNM staging system had better predictive accuracy than the TNM staging system and other clinicopathological characteristics alone. Thus, a novel predictive model incorporating the TNM staging system and dysbindin expression was developed. This nomogram showed better performance on prognostic accuracy than those of TNM staging system.

To our knowledge, the most pressing concern of patients with GAC is the duration of postoperative survival. To this end, we constructed this nomogram to predict of 3-year and 5-year overall survival. This nomogram could provide surgeons and patients with not only a general prognosis at the time of diagnosis but also information that can be used to select appropriate treatment. In addition, dysbindin expression could be used to classify patients within each stage into high- and low-risk groups and allow surgeons to identify potential candidates for systemic treatment to improve outcomes. Therefore, this nomogram may be a valid and useful tool for clinicians in routine clinical practice. Patients with GAC may choose to undergo systemic treatments to improve outcomes on the basis of this nomogram and their dysbindin expression levels.

Several classic clinical trials have indicated GAC patients derive a survival benefit from postoperative adjuvant chemotherapy, which has led to it being widely recommended as standard therapy, especially for stage II and III patients [31, 32]. However, there have been large variations in chemotherapy response and clinical outcomes [33]. Hence, identifying patients who likely to be sensitive to chemotherapy will not only improve outcomes but also reduce excessive toxicities. Currently, fluorouracil is generally used as a mainstream chemotherapeutic drug. In this study, we assessed the association between dysbindin expression and the OS of patients with stage II and III GAC who received fluorouracil-based postoperative advanced chemotherapy. The results suggested that patients with low level of dysbindin expression were more likely to derive a survival benefit from PACT than those with high dysbindin expression levels. This strongly indicated that dysbindin expression could be a promising predictive factor for the response to chemotherapy. To our knowledge, no study has reported the relationship between the expression of dysbindin and chemotherapy response. This new molecular feature of GAC may help surgeons select and manage candidates for fluorouracil-based chemotherapy.

In recent years, increasing attention has been paid to precise molecular tumor features in terms of survival prediction and the potential response to therapy. LncRNA MNX1-AS1 has been reported to be upregulated in gastric cancer, and its upregulation indicates poor prognosis [34]. Another lncRNA panel has been reported to predict the chemotherapy response of early-stage colorectal cancer [35]. Wang et al. reported that lncRNA-ROR regulated multidrug resistance genes and predicted poor prognosis in patients with gastric cancer [36]. To our knowledge, although these signatures showed promising results with regard to assessing the response to chemotherapy, these tests were mainly based on RT-PCR, which requires high technical proficiency and skilled operators. However, IHC has been widely applied in routine clinical tests, which provide not only quantitative analyses of target proteins but also their accurate cellular localization. Hence, we believe that the identification of dysbindin expression in GAC with IHC could be a stable and routine molecular profiling test that could be used to predict the response of GAC to chemotherapy.

In this study, we developed a novel nomogram including age, pathological differentiation, Lauren's classification, TNM stage and dysbindin expression. This nomogram showed a satisfactory predictive 
ability for OS, as reflected in the C-index value and AUC. More importantly, this nomogram retained its predictive value after internal bootstrap resampling and external validation in a cohort from another hospital, as recommended by statisticians [37]. We believed that the selection bias could be minimized with internal bootstrap resampling. Furthermore, the transferability and generalizability of this nomogram were confirmed in an independent external cohort. Thus, the newly developed model showed promising predictive value for individualized risk stratification and OS.

Several advantages of this nomogram were also observed. First, the predictions made using this nomogram could inform the initial postoperative OS assessment. GAC patients with a poor prognosis according to this nomogram may need more proactive treatment, which may not be limited to chemotherapy. Second, this nomogram could identify patients who are likely to be sensitive to fluorouracilbased chemotherapy based on individualized molecular features. Since chemotherapy is recommended for patients with advanced GAC, we think this is very important to avoid ineffective chemotherapy and identify the candidates who are likely to be sensitive to fluorouracil. Third, using this nomogram, the follow-up plan for patients with poor predicted prognosis could be formulated with consideration of that prediction. Clinicians may be able to provide more accurate prospective evaluations to these patients. Fourth, the specific characteristics incorporated in this nomogram were simple and readily available, and even the pathological factors were routinely obtained in clinical practice. Fifth, the decision curve constructed based on this nomogram may facilitate the assessment by clinicians of the net clinical benefits of treatment.

Although this nomogram showed promising predictive value, this study has several limitations. Firstly, its retrospective nature limited the study to some extent, and the number of patients polled was still relatively small. Secondly, the OS of patients was affected by not only GAC but also comorbidities, which were not reflected in this nomogram. It is complicated to categorize and quantify those variables. Third, this nomogram did not account for the impact of race on OS. All the participants enrolled in this study were Asian, which may limit the widespread applicability of the nomogram because of the different treatment strategies and possible variations due to differences among ethnicities.

In summary, this study showed that the expression level of dysbindin is an independent prognostic predictive risk factor in patients with GAC. The newly developed nomogram incorporating the TNM staging system and dysbindin expression showed promising prognostic predictive capacity after internally and externally validation. More importantly, dysbindin expression could be used to identify a subset of patients with advanced GAC who are likely to be sensitive to fluorouracil-based postoperative advanced chemotherapy. These findings may shed new light on individualized precision therapy for GAC. However, the clinical value of this nomogram still needs further confirmation in a prospective study.

\section{Abbreviations}

GAC: gastric adenocarcinoma; IHC: immunohistochemistry; TNM: tumor-node-metastasis; HR: hazard ratio; CI: confidence interval; PACT: postoperative adjuvant chemotherapy treatment; DCA: decision curve analysis; ROC: receiver operating characteristic.

\section{Supplementary Material}

Supplementary figures and tables. http://www.jcancer.org/v12p6740s1.pdf

\section{Acknowledgements}

This study was funded by the National Natural Science Foundation of China with grant/award numbers No. 81672319, No. 81903073, No. 82002740, and the Natural Science Foundation of Shaanxi Province with grant/award Numbers of 2020JQ-452 and 2020JQ-468.

\section{Competing Interests}

The authors have declared that no competing interest exists.

\section{References}

1. Siegel RL, Miller KD, Jemal A. Cancer statistics, 2020. CA: a cancer journal for clinicians. 2020; 70: 7-30.

2. Bray F, Ferlay J, Soerjomataram I, et al. Global cancer statistics 2018: GLOBOCAN estimates of incidence and mortality worldwide for 36 cancers in 185 countries. CA Cancer J Clin. 2018; 68: 394-424.

3. Thrift AP, El-Serag HB. Burden of Gastric Cancer. Clinical gastroenterology and hepatology : the official clinical practice journal of the American Gastroenterological Association. 2020; 18: 534-42.

4. Karimi P, Islami F, Anandasabapathy S, et al. Gastric cancer: descriptive epidemiology, risk factors, screening, and prevention. Cancer epidemiology, biomarkers \& prevention : a publication of the American Association for Cancer Research, cosponsored by the American Society of Preventive Oncology. 2014; 23: 700-13.

5. Shitara K, Morita S, Fujitani K, et al. Combination chemotherapy with S-1 plus cisplatin for gastric cancer that recurs after adjuvant chemotherapy with S-1: multi-institutional retrospective analysis. Gastric cancer : official journal of the International Gastric Cancer Association and the Japanese Gastric Cancer Association. 2012; 15: 245-51.

6. Wagner AD, Syn NL, Moehler M, et al. Chemotherapy for advanced gastric cancer. The Cochrane database of systematic reviews. 2017; 8: Cd004064.

7. De Vita F, Orditura M, Matano E, et al. A phase II study of biweekly oxaliplatin plus infusional 5-fluorouracil and folinic acid (FOLFOX-4) as first-line treatment of advanced gastric cancer patients. British journal of cancer. 2005; 92: 1644-9.

8. Kubota T. The role of S-1 in the treatment of gastric cancer. British journal of cancer. 2008; 98: 1301-4. 
9. Sasako M, Sakuramoto S, Katai H, et al. Five-year outcomes of a randomized phase III trial comparing adjuvant chemotherapy with S-1 versus surgery alone in stage II or III gastric cancer. J Clin Oncol. 2011; 29: 4387-93.

10. Choi AH, Kim J, Chao J. Perioperative chemotherapy for resectable gastric cancer: MAGIC and beyond. World journal of gastroenterology. 2015; 21: 7343-8.

11. Ilson DH. Advances in the treatment of gastric cancer. Current opinion in gastroenterology. 2018; 34: 465-8.

12. Wittekind C. The development of the TNM classification of gastric cancer. Pathology international. 2015; 65: 399-403.

13. Ilhan E, Ureyen O, Meral UM. Ongoing problems concerning 7(th) TNM Staging System and Proposals for 8(th) TNM Staging System of Gastric Cancer. Przeglad gastroenterologiczny. 2016; 11: 223-5.

14. Ghiani CA, Dell'Angelica EC. Dysbindin-containing complexes and their proposed functions in brain: from zero to (too) many in a decade. ASN neuro. 2011; 3: e00058

15. Zhu D, Zheng S, Fang C, et al. Dysbindin promotes pancreatic ductal adenocarcinoma metastasis by activating NF-kappaB/MDM2 via miR-342-3p. Cancer letters. 2020; 477: 107-21.

16. Fang C, Guo X, Lv X, et al. Dysbindin promotes progression of pancreatic ductal adenocarcinoma via direct activation of PI3K. J Mol Cell Biol. 2017; 9: 504-15.

17. Lv X, Guo X, Ru Y, et al. Dysbindin facilitates invasion and metastasis by promoting phosphorylation of ERK in epithelial ovarian cancer. J Cancer. 2020; 11: 2821-9.

18. Johnston FM, Beckman M. Updates on Management of Gastric Cancer. Current oncology reports. 2019; 21: 67

19. Lauren P. THE TWO HISTOLOGICAL MAIN TYPES OF GASTRIC CARCINOMA: DIFFUSE AND SO-CALLED INTESTINAL-TYPE CARCINOMA. AN ATTEMPT AT A HISTO-CLINICAL CLASSIFICATION. Acta pathologica et microbiologica Scandinavica. 1965; 64: 31-49.

20. Washington K. 7th edition of the AJCC cancer staging manual: stomach. Annals of surgical oncology. 2010; 17: 3077-9.

21. Shou ZX, Jin X, Zhao ZS. Upregulated expression of ADAM17 is a prognostic marker for patients with gastric cancer. Annals of surgery. 2012; 256: 1014-22.

22. Cao Y, Liu H, Li H, et al. Association of O6-Methylguanine-DNA Methyltransferase Protein Expression With Postoperative Prognosis and Adjuvant Chemotherapeutic Benefits Among Patients With Stage II or III Gastric Cancer. JAMA Surg. 2017; 152: e173120.

23. Zhang H, Liu H, Shen Z, et al. Tumor-infiltrating Neutrophils is Prognostic and Predictive for Postoperative Adjuvant Chemotherapy Benefit in Patients With Gastric Cancer. Ann Surg. 2018; 267: 311-8.

24. Jiang Y, Xie J, Han Z, et al. Immunomarker Support Vector Machine Classifier for Prediction of Gastric Cancer Survival and Adjuvant Chemotherapeutic Benefit. Clin Cancer Res. 2018; 24: 5574-84.

25. Jiang Y, Li T, Liang X, et al. Association of Adjuvant Chemotherapy With Survival in Patients With Stage II or III Gastric Cancer. JAMA Surg. 2017; 152: e171087.

26. Vickers AJ, Cronin AM, Elkin EB, et al. Extensions to decision curve analysis, a novel method for evaluating diagnostic tests, prediction models and molecular markers. BMC Med Inform Decis Mak. 2008; 8: 53.

27. Xu S, Cao S, Geng J, et al. High prognostic nutritional index (PNI) as a positive prognostic indicator for non-small cell lung cancer patients with bone metastasis. The clinical respiratory journal. 2020; 15: 225-31

28. Li R, Liu X, Zhou XJ, et al. Identification of a Prognostic Model Based on Immune-Related Genes of Lung Squamous Cell Carcinoma. Frontiers in oncology. 2020; 10: 1588 .

29. Li Y, Liu W, Zhao L, et al. Nomograms predicting Overall Survival and Cancer-specific Survival for Synchronous Colorectal Liver-limited Metastasis. Journal of Cancer. 2020; 11: 6213-25.

30. Jin LP, Liu T, Meng FQ, et al. Prognosis prediction model based on competing endogenous RNAs for recurrence of colon adenocarcinoma. BMC cancer. 2020; 20: 968 .

31. Smyth EC, Nilsson M, Grabsch HI, et al. Gastric cancer. Lancet (London, England). 2020; 396: 635-48.

32. Zhu Z, Gong YB, Xu HM. Neoadjuvant therapy strategies for advanced gastric cancer: Current innovations and future challenges. Chronic diseases and translational medicine. 2020; 6: 147-57.

33. Gambardella V, Fleitas T, Tarazona N, et al. Precision Medicine to Treat Advanced Gastroesophageal Adenocarcinoma: A Work in Progress. Journal of clinical medicine. 2020; 9: 3049 .

34. Shuai $Y$, Ma Z, Liu $W$, et al. TEAD4 modulated LncRNA MNX1-AS1 contributes to gastric cancer progression partly through suppressing BTG2 and activating BCL2. Molecular cancer. 2020; 19: 6 .

35. Zhou R, Sun H, Zheng S, et al. A stroma-related lncRNA panel for predicting recurrence and adjuvant chemotherapy benefit in patients with early-stage colon cancer. Journal of cellular and molecular medicine. 2020; 24: 3229-41.

36. Wang S, Chen W, Yu H, et al. IncRNA ROR Promotes Gastric Cancer Drug Resistance. Cancer control : journal of the Moffitt Cancer Center. 2020; 27: 1073274820904694

37. Steyerberg EW, Vergouwe Y. Towards better clinical prediction models: seven steps for development and an ABCD for validation. European heart journal. 2014; 35: 1925-31. 